\title{
EVALUATION OF SEROUS EFFUSIONS USING AgNOR COUNT- A STUDY IN A TERTIARY CARE CENTRE OF SAHARANPUR
}

\author{
Rachna Sharma ${ }^{1}$, Vinay Sharma², Neetu Goyal ${ }^{3}$
}

${ }_{1}^{1}$ Assistant Professor, Department of Pathology, SMMH Medical College, Saharanpur, Uttar Pradesh, India.

${ }^{2}$ Assistant Professor, Department of Pathology, Venkateshwara Institute of Medical Sciences, Gajraula, Uttar Pradesh, India.

${ }^{3}$ Assistant Professor, Department of Pathology, SMMH Medical College, Saharanpur, Uttar Pradesh, India.

\begin{abstract}
BACKGROUND

Abnormalities of nucleolar morphology are constant features of cancer cells. The importance of the nucleolus for the diagnosis of malignancy has been reconsidered after the observation that structural and morphological reorganisation is a consequence of the redistribution of the fibrillar components within the nucleolus. The nucleolar fibrillar components (fibrillar centre and ass ociated dense fibrillar component) are the sites where ribosomal genes are located during interphase. They therefore represent the interphase counterpart of metaphase Nucleolar Organizer Regions (NOR's). The use of this parameter relative to interphase, NOR distribution has become of great importance after the finding that interphasic NOR's can be clearly visualised at the light microscopic level using a silver reaction, which stains the acidic proteins of the NOR's (AgNOR Proteins) on routinely prepared histo- and cytological samples. By means of this technique, silver-stained interphasic NOR's has been used for the diagnosis of malignancy of numerous neoplastic lesions. Cancer cells in fact have a greater number of silver-stained NOR's than the corresponding benign cells. The diagnosis of malignancy in cytologic preparations of pleural and peritoneal effusions is not always easily performed. Interest therefore has focussed on identifying reliable methods of supplementing conventional cytological methods to differentiate malignant cells from benign cells. Recently, AgNOR's (Silver Staining of Nucleolar Organizer Regions) have come to limelight. NOR's are being increasingly used in diagnosing a variety of tumours.
\end{abstract}

Aims and Objectives:

The objectives of the present study were

1. To identify reliable methods of supplementing conventional cytological methods to differentiate malignant cells from benign cells in serous effusions.

2. To study various serous effusions by conventional cytological methods.

3. To differentiate benign and malignant serous effusions on the basis of conventional cytological methods.

4. To study AgNOR count in differentiating malignant cells from benign cells in serous effusions and

5. To find out the utility of AgNOR counts in malignant vs benign cell differentiation in fluids.

\section{MATERIALS AND METHODS}

This is a descriptive study. We studied 65 cases of serous effusions over a period of one year from December 2016 - December 2017. Samples for this study were collected from various outpatients and inpatients admitted in Medicine and Surgical wards.

Staining and counting procedure.

1. Fluids collected were subjected to-

- Pap staining.

- 'AgNOR' staining.

2. Cytopathological evaluation of smears was done using Papanicolaou staining and further differentiation using AgNOR count.

3. The number of AgNOR's present in each nucleus were counted. Two hundred nuclei were taken into account. The mean AgNOR count of the specimen was then calculated. The AgNOR's were visualised using oil immersion objective and to avoid biased results, counts were done by two different observers.

\section{RESULTS}

A total of 43 cases (66.15\%) of benign fluids were examined. Low mean AgNOR counts (less than 4 ) were seen in 41 cases (95.35\%), whereas 2 (4.65\%) benign cases had high (more than 4) mean AgNOR counts. Of the 22 (33.85\%) malignant fluids encountered, low mean AgNOR counts (less than 4) were observed only in one case with a percentage of 4.54\%, whereas high mean AgNOR counts were observed in 21 cases with a percentage of $95.46 \%$. Cytologically, all the malignant cases 22 (100\%) were diagnosed as adenocarcinoma with mean AgNOR counts as in previous paragraph.

\section{CONCLUSION}

The mean AgNOR counts have a definite role in differentiating benign from malignant effusions. They can be used as supportive test to increase the value of routinely used Papanicolaou staining methods.

\section{KEY WORDS}

AgNOR, Serous Effusion, Conventional Cytology.

HOW TO CITE THIS ARTICLE: Sharma R, Sharma V, Goyal N. Evaluation of serous effusions using AgNOR count- a study in a tertiary care centre of Saharanpur. J. Evolution Med. Dent. Sci. 2018;7(47):5057-5062, DOI: 10.14260/jemds/2018/1125 
'Financial or Other Competing Interest': None.

Submission 04-10-2018, Peer Review 02-11-2018,

Acceptance 09-11-2018, Published 19-11-2018.

Corresponding Author:

Dr. Neetu Goyal,

C/o. Dr. A. K. Jain,

Court Road, Behind Petrol Pump,

Opp. ITO, Saharanpur, Uttar Pradesh, India.

E-mail: neetuthedoc@gmail.com

DOI: $10.14260 /$ jemds $/ 2018 / 1125$

\section{(c) $(1) Ð$}

\section{BACKGROUND}

Accumulation of fluid in a serous cavity in excess of the normal small amount is referred to as an effusion. The likely benign causes of effusions included in the pleura arecongestive heart failure, pulmonary infarcts, inflammatory processes including viral pneumonia and tuberculosis and cirrhosis of liver causing peritoneal effusion.

Aspiration of serous cavities is a simple and relatively non-invasive technique to achieve a diagnosis. In malignant conditions, accumulation of serous fluid is more commonly due to secondary involvement of the serous membranes than the primary malignant tumour of the mesothelium-malignant mesothelioma. The diagnosis of malignancy in cytologic preparations of pleural and peritoneal effusions is not always easily performed. Malignant cells in effusions take on some characteristics of in-vitro cultural cells: in particular they frequently lose their relationship with surrounding structures.

Abnormalities of nucleolar morphology are constant features of cancer cells. The importance of the nucleolus for the diagnosis of malignancy has been reconsidered after the observation that structural and morphological reorganisation is a consequence of the redistribution of the fibrillar components within the nucleolus. The nucleolar fibrillar components (fibrillar centre and associated dense fibrillar component) are the sites where ribosomal genes are located during interphase. They therefore represent the interphase counterpart of metaphase nucleolar organizer regions (NOR's)

The use of this parameter relative to interphase NOR distribution has become of great importance after the finding that interphasic NOR's can be clearly visualised at the light microscopic level using a silver reaction, which stains the acidic proteins of the NOR's (Ag-NOR Proteins) on routinely prepared histo- and cytological samples. By means of this technique, the quantity of silver-stained interphasic NOR's has been used for the diagnosis of malignancy of numerous neoplastic lesions. Cancer cell share in fact have a greater number of silver-stained NOR's than the corresponding benign cells.

\section{MATERIALS AND METHODS}

\section{Study Design}

The present descriptive study was undertaken at Department of Pathology of a tertiary care centre, conducted over a period of 1 year from December 2016 to December 2017.

\section{Study Population}

A total of 65 cases of body effusions were included in the present study. Samples for this study were collected from various outpatients and inpatients admitted in Medicine and Surgical wards of our institution.

\section{Collection of Data}

Clinical data such as site of effusion, radiological findings and provisional clinical diagnosis were obtained from the patient's medical records.

\section{Sample Evaluation}

Collection of Sample: - Samples from patients for this study were collected under strict aseptic precautions, were processed as soon as possible. $10 \%$ formalin in equal amount was used as preservative for fluid. Fluid so collected were subjected to-

- $\quad$ Pap staining.

- 'AgNOR' staining.

\section{Improved Silver Technique for AGNOR Staining- Silver Colloid Solution:}

- $\quad 2 \mathrm{~g} / \mathrm{dL}$ gelatin dissolved in $1 \mathrm{~g} / \mathrm{dL}$ aqueous formic acid.

- The above-mentioned solution was mixed just before staining in a ratio of $1: 2$ volumes with a $20 \mathrm{gm} / \mathrm{dL}$ aqueous silver nitrate solution.

\section{Technique}

1. Fix the smear in 3: 1 ethanol-acetic acid solution for thirty minutes.

2. Rinse briefly in absolute alcohol for 5 seconds.

3. Rinse briefly in $\mathbf{7 0}$ percent alcohol for $\mathbf{5}$ seconds.

4. Rinse in running water for ten minutes and then washed three times in deionized distilled water.

5. Slides are then placed in freshly prepared staining solution for 30 minutes at room temperature in the dark.

6. Sides are then washed 6 times with distilled water.

7. Dehydrated by passing three or four baths of absolute alcohol.

8. Cleared in xylene.

9. Mounted with DPX mountant.

\section{RESULTS}

AgNOR's appeared within nuclei as dark brownish dots of varying sizes against yellow background.

\section{Counting Procedure}

The number of AgNOR's present in each nuclei are counted. Two hundred nuclei are taken into account. The mean AgNOR count of the specimen is then calculated. The AgNOR's were visualised using oil immersion objective and to avoid biased results the counts were done by two different observers.

Cytopathological evaluation of smears was done using Papanicolaou staining and further differentiation using AgNOR count.

\section{Statistical Analysis}

The data was entered in MS-Excel and analysed using EpiInfo 7 software. Frequencies and means of finding were calculated and appropriate test of significance (Fisher Exact Test) were applied.

The present study comprised of Sixty five (65) cases of different body effusions over a period of one year from Dec. 2016 to Dec. 2017, received in the Department of Pathology. SMMH Medical College, Saharanpur, collected from various outpatients and inpatients admitted in Medicine and Surgical wards of this medical college, hospital.

Following observations were made. 


\begin{tabular}{|c|c|c|c|}
\hline SI. No. & Age in Yrs. & No. of Cases & (\%) \\
\hline 1. & $0-15$ & 02 & $3.07 \%$ \\
2. & $16-30$ & 13 & $20.00 \%$ \\
3. & $31-45$ & 09 & $13.85 \%$ \\
4. & $46-60$ & 28 & $43.07 \%$ \\
5. & $61-75$ & 12 & $18.46 \%$ \\
6. & $76-90$ & 01 & $1.55 \%$ \\
\hline \multicolumn{2}{|c|}{ Table 1. Age-Wise distribution of Cases Analysed } \\
\hline
\end{tabular}

Table 1. Shows age-wise distribution of total cases analysed.

\begin{tabular}{|c|c|c|c|}
\hline SI. No. & Sex & No. of Cases & (\%) \\
\hline 1. & Females & 34 & $52.31 \%$ \\
2. & Males & 31 & $47.69 \%$ \\
\hline \multicolumn{4}{|c|}{ Table 2. Sex-Wise distribution of Cases Analysed } \\
\hline
\end{tabular}

Table 2. As is evident from the table, most of the patients were females $34(52.31 \%)$ and rest $31(47.69 \%)$ were males.

\begin{tabular}{|c|c|c|c|}
\hline SI. No. & Type of Fluid & No. of Cases & (\%) \\
\hline 1. & Peritoneal Fluid & 38 & $58.46 \%$ \\
2. & Pleural Fluid & 23 & $35.38 \%$ \\
3. & Pericardial Fluid & 04 & $06.16 \%$ \\
4. & Synovial Fluid & - & - \\
5. & Others & - & - \\
\hline \multicolumn{3}{|c|}{ Table 3. Different types of Body Fluids/Effusions Analysed } \\
\hline
\end{tabular}

Table 3. Shows different types of fluids analysed in the present study. In all Ascitic fluids (peritoneal fluids) were 38 (58.46\%), pleural fluid were $23(35.38 \%)$ and only 4 cases $(6.16 \%)$ of pericardial fluid were encountered and analysed.

\begin{tabular}{|c|c|c|c|}
\hline SI. No. & Type of Fluid & No. of Cases & (\%) \\
\hline 1. & Transudate & 36 & $55.38 \%$ \\
2. & Exudate & 29 & $44.62 \%$ \\
\hline \multicolumn{3}{|c|}{ Stuble 4. Distribution of Various Fluids Encountered in the } \\
\hline
\end{tabular}

Table 4. Shows distribution of total cases on the basis of their protein contents. Mostly 36 (55.38\%) fluids were transudates and rest 29 (44.62\%) were exudates.

\begin{tabular}{|c|c|c|c|}
\hline SI. No. & Nature of Effusions & No. of Cases & \% \\
\hline 1. & Benign & 43 & $66.15 \%$ \\
2. & Malignant & 22 & $33.85 \%$ \\
\hline \multicolumn{3}{|c|}{ Table 5. Benign/ Malignant Nature Wise distribution of } \\
\hline \multicolumn{3}{|c|}{ Cases Anlysed } \\
\hline
\end{tabular}

Table 5. As the table shows, in the entitled study there were 43 cases $(66.15 \%)$ of benign effusions and malignant effusions were 22 (33.85\%).

\begin{tabular}{|c|c|c|c|}
\hline SI. No. & Cytological Diagnosis & No. of Cases & $\%$ \\
\hline 1. & Benign & 43 & $66.15 \%$ \\
& Malignant & & \\
2. & (a) Adenocarcinoma & 22 & $33.85 \%$ \\
& (b) Squamous cell & 22 & $100 \%$ \\
& carcinoma & - & - \\
& (c) Lymphoma & - & - \\
& (d) Melanoma & - & - \\
& (e) Others & - & - \\
\hline \multicolumn{2}{|c|}{ Table 6. Cytological Diagnosis of Different Body Fluids } \\
\hline
\end{tabular}

Table 6. Shows cytodiagnosis in total cases analysed. Out of 65 cases, 43 cases (66.15\%) were benign and 22 (33.85\%) cases were diagnosed as malignant as regard to malignant effusions. Cytologically, all cases were adenocarcinomas with an incidence of $100 \%$. No case of squamous cell carcinoma, lymphoma and melanomas were seen.

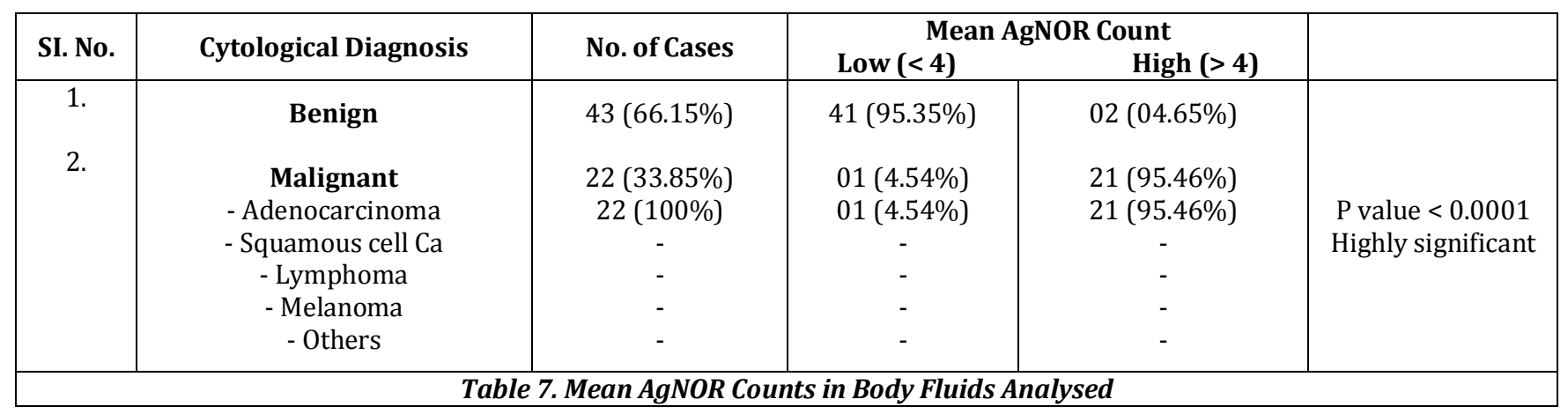

Table 7. Shows analysis of body fluids and their evaluation on the basis of AgNOR counts. A total of 43 cases $(66.15 \%)$ of benign fluids were encountered with low mean AgNOR counts (less than 4) in 41 cases (95.35\%), whereas 2 (4.65\%) benign cases had high (more than 4 ) mean AgNOR counts.

In all, 22 (33.85\%) malignant fluids were encountered. Low mean AgNOR counts (less than 4) were observed only in one case with a percentage of $4.54 \%$. Whereas high mean AgNOR counts were observed in 21 cases with a percentage of $95.46 \%$.
Cytologically, all the malignant cases 22 (100\%) were diagnosed as adenocarcinoma with mean AgNOR counts as in previous paragraph.

In the above table, Fisher exact test was applied, and pvalue was $<0.0001$ which is highly significant. This tells that there is significant association between malignancy and high AgNOR count in the present study.

(Fisher exact test is test of significance for categorical). 


\section{DISCUSSION}

The present work on Silver staining of "Nucleolar Organiser Region's" (AgNOR's) in body effusions has been carried out in Pathology Department of SMMH Medical College, Saharanpur, to evaluate their role in differentiating benign and malignant effusions. A total of sixty-five body fluids as effusions including benign and malignant were analysed.

Howell et al (1975) ${ }^{[1]}$ pointed out an important and surprising feature of NOR's. They pointed out that some of the NOR's are completely negative for Ag-As staining. The property of NOR's to stain with Ag-As staining depends also upon the physiological activity of NOR's apart from presence of rDNA. The silver staining of NOR's in chromosomes has drawn attention on few important points-

1. Only active NOR's can be stained (Miller et al, 1978).[2]

2. Evidence to date supports the view that each individual has a characteristic or model number of AgNOR's (Bloom and Good Pasture, 1976).[3] This raises the prospect of determining the familial transmission of individual acrocentric chromosomes.

3. The silver positive material is located around the proposed NOR site, but never directly on the chromatin segment (Schwarzacher et al, 1983).[4]

4. Tendency of acrocentric regions to associate through satellite regions randomly. The AgNOR technique demonstrate the presence of physical connections indicating that the respective chromosomes participated together in nuclear organisations.

The distribution of NOR's in cytologic preparations of human serous effusions with the aim of differentiating malignant and non-malignant reactive cells was studied by Derenzini et al (1989).[5] He suggested that malignant cells were characterised by an irregularly scattered distribution of AgNOR's were determined by NOR's and by a heterogeneity in the size of NOR's in addition to an increased number of NOR's.

Carrillo et al (1994)[6] in thirty eight pleural and peritoneal effusions from patients with malignant solid tumours. Cytologically, 13 effusions were benign, 23 malignant and 2 indeterminate. The mean AgNOR counts for benign, malignant and indeterminate specimens were 2.99, 3.94 and 3.50 respectively.

Ayres et al (1988)[7] observed a mean AgNOR value of 1.04 for normal and 1.75 for reactive mesothelium in biopsy samples.

In a similar study, Trevisan et al (1993) found a mean count of 1.64 for normal reactive mesothelium. Sujathan et al $(1996)^{[8]}$ studied one hundred aspirated samples of benign and malignant effusions using the one-step silver staining method. AgNOR study be Sujathan et al (1996) concluded that in malignant cells the mean AgNOR count was 4.72 and the AgNOR's were irregular is shape, while in benign mesothelial cells AgNOR's were comparatively larger, single dots with a mean count of 1.92, cytologically atypical samples, five were in the malignant range, the others were within benign limits. In malignant lesions, the highest mean AgNOR count was seen in Lymphoma (5.44) and lowest observed in adenocarcinoma (4.57).

The results of study of Sujathan et al (1996) ${ }^{[8]}$ suggest that the AgNOR method can be valuable as an additional diagnostic tool for use on ascitic or pleural fluid.
Peritoneal fluids constituted as the largest number of effusions. Pleural fluid and pericardial fluid cases were small in number. Similar types of fluids have been included and analysed by the studies of Derenzini et al (1989)[5] and Carrillo et al (1994).[6] Surprisingly, they have not included pericardial effusions in their study.

Most of the body fluids were transudates and exudates were small in number. Present study showed mostly benign effusions as compared to malignant effusions. The ratio was $2: 1$. In the study of Carrillo et al (1994),[6] the ratio of benignto-malignant was reversed, i.e. 13 were benign and 23 were malignant, whereas 2 cases were indeterminate. Sujathan et al (1989)[8] in their study again showed a more or less increased preponderance of malignant cases over benign with a ratio of (1.2: 1). The present reversal of ratio in our study can be explained on account of, since it was a small study and the time period again was small.

Cytodiagnosis of malignant effusions showed adenocarcinoma. Squamous cell carcinoma, lymphoma and melanomas were not observed. Similar studies have been conducted by Derenzini et al (1989),[5] who analysed 100 cases of malignant effusions that included 45 metastatic pleural effusions and 25 peritoneal effusions as metastatic adenocarcinoma. This study also included mesothelioma cases and reactive pleural effusions. In another study carried out by Carrillo et al (1994)[6] has also reported adenocarcinomas along with squamous cell carcinoma and a fair number of cases of melanomas. In the study of Sujathan et al (1989),[8] in the analysis of 55 cases included 45 adenocarcinoma along with cases of lymphoma and melanomas and also anaplastic carcinoma.

So our findings are in accordance with the study by Derenzini et al (1989),[5] Carrillo et al (1994)[6] and Sujathan et al (1989) ${ }^{[8]}$ as regards to maximum number, 01 case of adenocarcinoma.

The mean AgNOR counts measured in different body fluids showed a mean AgNOR count of less than 4 in benign effusions and mean AgNOR counts of more than 4 in malignant effusions. Similar findings about mean AgNOR counts have been observed by Carrillo et al (1994),[6] Ayres et al (1988) ${ }^{[7]}$ and Sujathan et al (1989).[8]

Carrillo et al (1994)[6] has showed a lower mean AgNOR count value of less than four per nucleus in benign effusions. Surprisingly, some of the malignant cases had also been reported having less than four counts, whereas majority of malignant effusions had mean AgNOR count more than four. No satisfactory explanation has been offered by the author for this discrepancy.

Ayres et al (1988)[7] in his study has reported mean AgNOR value of 1.04 for normal and mean AgNOR value of 1.75 for reactive mesothelium, similarly Trevisan et al (1993) has reported mean count of 1.64 for normal reactive mesothelium. When compared our findings are in accordance with the findings of Sujathan et al (1989). [8]

While differentiating benign effusions from malignant effusions some workers have taken into account AgNOR dot sizes, larger dots or smaller dots. In malignant forms, dot size has been smaller as compared to dot size in benign effusions. In one single case of moderately differentiated adenocarcinoma effusion, the AgNOR dots were very large and were lesser in number. It might be that the size was definitely increased or it was due to groups of nucleolar 
organizer regions which could not be visualised separately at provided optical level. Similar relationship of AgNOR dot sizes and degree of malignancy has been stressed upon by Derenzini et al (1987)[9] and Crocker et al (1988).[10] Somewhat opposite observations as regard to AgNOR dots have been reported by Crocker and Egan (1988),[11] who have reported that AgNOR dot size increases with increasing degree of malignancy. Here, our findings are different from the above observations.

It was noticed that NOR dots could be easily visualised within the nucleoli in most of the cases. Similar observational views have been put forward by Crocker et al (1989) ${ }^{[12]}$ who was of the opinion that AgNOR dots are well seen lying within the nucleoli in the tumour cells in contrast to resting cells where they are not easily seen.

The mean AgNOR counts increases significantly as we move from well-differentiated carcinomas to poorly differentiated carcinomas. These findings can be explained on the basis of the speculations given by Crocker et al (1988),[10] who observed a linear correlation between the mean numbers of AgNOR per nucleolus and percentage of S phase cell and suggested that the numbers on AgNOR's in a lymphoma may be related to the dividing fraction of the cell rather than to the ploidy alone.

Jan-Mohamed (1989)[13] stated that AgNOR count may be increased due to increased number of chromosomes or increased cell turnover. Underwood and Giri (1988) ${ }^{[14]}$ states that increase in AgNOR count is not an absolute increase in the number, but rather an index of dispersion.

Different cytogenetics explained differently the causes of increased NOR counts in malignancy. It may be due to increase in the chromosome number, increasing the chromosome arms containing NOR's (Schwarzacher et al, 1988),[4] gene amplification (Roberts et al, 1987),[15] but it is most significantly related to proliferation (Cairns et al 1989).[16]

The AgNOR dots have been utilised for differentiating various histological types of tumours in biopsy specimens as in diagnosing malignancies of breast (Smith et al, 1988). Endometrium (Hansen et al, 1990),[17] cervix (Egan et al, $1990,[18]$ ), ovaries (Griffiths et al, 1989),[19] pleural mesothelium (Ayres et al, 1988)[7] and skin (Egan MJ et al, 1988).[20]

The value of AgNOR dots in relation to differentiate various types of malignancies in effusions has been evaluated by Ayres et al (1988), Derenzini et al (1989) ${ }^{[5]}$ and Egan et al (1990).[18]

Thus, it could he concluded that though NOR's have a definite role in differentiating benign from malignant effusions, they do not supersede the value of routinely used Papanicolaou staining methods as a considerable overlap is seen between the various grades of malignancy. Kodousek $\mathrm{R}$ and Dusek J (1991) ${ }^{[21]}$ also points out that AgNOR technique does not exceed the value of routinely stained smears of good quality. However, in spite of these limitation, AgNOR staining methods seem to represent a valuable contribution as early when a cytologic diagnosis is difficult.

The present study shows significant association between malignancy and high AgNOR counts. The results of the study cannot be generalised due to a small sample size.

The AgNOR count if combined with other cell proliferation indices such as DNA flow cytometry, Ki-67 immunohistochemistry can lead to better understanding of neoplasms and then AgNOR counts may also assist in the grading of neoplasm and their prognosis.

\section{CONCLUSION}

Although, the mean AgNOR counts have a definite role in differentiating benign from malignant effusions, they do not however supersede the value of routinely used Papanicolaou staining methods, but definitely have a supportive role.

The clinical implication of AgNOR's determination in the evaluation of serosal effusions remains supportive. However, above study indicates that AgNOR counts have supportive practical value which can be utilised in differentiating malignant effusions from benign ones, when AgNOR findings are correlated with conventional cytological findings, clinical findings and other laboratory parameters. However, an effort should be directed towards better standardisation of techniques and counting of AgNOR's.

Also, such studies should be conducted on larger number of patients, so that the results can be generalised and baseline data for further research can be established.

\section{REFERENCES}

[1] Howell WM, Denton TE, Diamond JR. Differential staining of the satellite regions of human acrocentric chromosomes. Experimentia 1975;31(2):260-2.

[2] Miller DA, Breg WR, Warburton D, et al. Regulation of rRNA gene expression in a human familial 14 patient marker chromosomes. Hum Genet 1978;43(3):289-97.

[3] Bloom SE, Goodpasture C. An improved technique for selective silver staining of nucleolar organizer region in human chromosomes. Hum Genet 1976;34(2):199206.

[4] Schwarzacher HG, Wachtler F. Nucloelar organizer regions and nucleoli. Hum Genet 1983;63(2):89-99.

[5] Derenzini M, Nardi F, Farabegoli F, et al. Distribution of silver-stained interphase nucleolar organizer regions as a parameter to distinguish neoplastic from non-neoplastic and reactive cells in human effusions. Acta Cytologica 1989;33(4):491-8.

[6] Carrillo R, Sneige N, EI-Naggar AK. Interphase nucleolar organizer regions in the evaluation of serosal cavity effusions. Acta Cytologica 1994;38(3):367-72.

[7] Ayres JG, Crocker JG, Skilbeck NQ. Differentiation of malignant from normal and reactive mesothelial cells by argyrophil technique for nucleolar organizer region associated proteins. Thorax 1988;43(5):366-70.

[8] Sujathan K., Kannan S, Pillai KR, et al. Significance of AgNOR count in differentiating malignant cells from reactive mesothelial cells in serous effusions. Acta Cytologica 1996;40(4):724-8.

[9] Derenzini M, Betts CM, Eusebi V, et al. Distribution of interphase nucleolar organizer regions \& diagnosis of malignancy. The Lancet 1987;2(8553):286.

[10] Crocker J, McGovern J. Nucleolar organizer regions in normal, cirrhotic and carcinomatous livers. J Clin Pathol 1988;41(10):1044-8.

[11] Crocker J, Egan MJ. Correlation between NOR sizes and numbers in non-Hodgkin's lymphomas. J Pathol 1988;156(3):233-9. 
[12] Crocker J, Boldy DA, Egan MJ. How should we count AgNOR's - proposals for a standardized approach? Journal of Pathology 1989;158(3):185-8.

[13] Jan-Mohamed RM, Armstrong SJ, Crocker J, et al. Relationship between number of interphase NOR`s and NOR bearing chromosomes in Non-Hodgkin`s lymphoma. J Pathol 1989;158(1):3-7.

[14] Underwood JC, Giri DD. Nucleolar organizer regions as diagnostic discriminants for malignancy (editorial). J Pathol 1988;155(2):95-6.

[15] Roberts C, Brasch J, Tattersall MHN. Ribosomal RNA gene amplification: a selective advantage in tissue culture. Cancer Genet \& Cytogenet 1987;29(1):119-27.

[16] Cairns P, Suarez V, Newman J, et al. Nucleolar organizer regions in transitional cell tumours of the bladder. Arch Pathol Lab Med 1989;113(11):1250-2.

[17] Hansen A, Ostergard B. AgNOR counts in intraendometrial neoplasia. J Clin Pathol 1990;43 (6):518-9.
[18] Egan M, Freeth M, Crocker J. Relationship between intraepithelial neoplasia of cervix and the size and number of nucleolar organizer regions. Gynaecol Oncol 1990;36(1):30-3.

[19] Griffith AP, Butler CW, Roberts P, et al. Silver-stained structures (AgNOR's), their dependence on tissue fixation and absence of prognostic relevance in rectal adenocarcinoma. J Path 1989;159(2):121-7.

[20] Egan MJ, Raafat F, Crocker J, et al. Nucleolar organizer regions in fibrous proliferations of childhood and infantile fibrosarcoma. J Clin Pathol 1988;41(1):31-3.

[21] Kodousek R, Dusek J. Demonstration of the nucleolar organizer region by silver staining (AgNOR method) in research and histopathological practice. Acta Uni Palacki Olonuic Fac Med 1991;131:9-37. 